\title{
Detection of rifampin resistance patterns in Mycobacterium tuberculosis strains isolated in Iran by polymerase chain reaction- single-strand conformation polymorphism and direct sequencing methods
}

\author{
Bahram Nasr Isfahani ${ }^{+}$, Akbar Tavakoli, Mansoor Salehi*, Mehdi Tazhibi** \\ Medical Microbiology Department *Molecular Biology Department, School of Medicine \\ **School of Health, Isfahan University of Medical Sciences, Isfahan, Iran
}

Mutations in the rpoB locus confer conformational changes leading to defective binding of rifampin (RIF) to rpoB and consequently resistance in Mycobacterium tuberculosis. Polymerase chain reaction-single-strand conformation polymorphism (PCR-SSCP) was established as a rapid screening test for the detection of mutations in the $\mathrm{rpoB}$ gene, and direct sequencing has been unambiguously applied to characterize mutations.

A total of 37 of Iranian isolates of $\mathrm{M}$. tuberculosis, 16 sensitive and 21 resistant to RIF, were used in this study. A 193-bp region of the rpoB gene was amplified and PCR-SSCP patterns were determined by electrophoresis in 10\% acrylamide gel and silver staining. Also, 21 samples of 193-bp rpoB amplicons with different PCR-SSCP patterns from RIFr and 10 from RIFs were sequenced.

Seven distinguishable PCR-SSCP patterns were recognized in the 21 Iranian RIFr strains, while 15 out of 16 RIFs isolates demonstrated PCR-SSCP banding patterns similar to that of sensitive standard strain H37Rv. However one of the sensitive isolates demonstrated a different pattern. There were seen six different mutations in the amplified region of rpoB gene: codon 516(GAC/GTC), 523(GGG/GGT), 526(CAC/TAC), 531(TCG/TTG), 511(CTG/TTG), and $512(A G C / T C G)$.

This study demonstrated the high specificity (93.8\%) and sensitivity (95.2\%) of PCR-SSCP method for detection of mutation in rpoB gene; $85.7 \%$ of RIFr strains showed a single mutation and $14.3 \%$ had no mutations. Three strains showed mutations caused polymorphism. Our data support the common notion that rifampin resistance genotypes are generally present mutations in codons 531 and 526, most frequently found in M. tuberculosis populations regardless of geographic origin.

Key words: Mycobacterium tuberculosis - rpoB gene mutation - polymerase chain reaction-single-strand conformation polymorphism - direct sequencing

Mycobacteria are responsible for considerable human morbidity and mortality worldwide (Bahremand 1992). In the last decade, tuberculosis (TB) has reemerged as one of the leading causes of death with nearly 3 million deaths annually (Bloom \& Murray 1992). The emergence of AIDS and decline of socio-economic standards contribute to the disease's resurgence in industrialized countries (Frieden et al. 1993, Ashok et al. 1998).

A more alarming aspect of tuberculosis is the emergence of multidrug-resistant strains of $M$. tuberculosis (MDRTB) that is a widespread phenomenon (Snider \& Roper 1992, Frieden et al. 1993, Iseman 1993, Kato-Maeda et al. 1999). The common finding has been a high rate of primary resistance to isoniazid (INH) and to the combination of isoniazid and rifampin (RIF) (Garcia-Garcia 2000) and a high rate of resistant (17.2\%) to all the four commonly used antimycobacterial agents for treatment and

Financial support: Isfahan University of Medical Sciences

${ }^{+}$Corresponding author: nasr@hlth.mui.ac.ir

Received 16 January 2006

Accepted 12 July 2006 prophylaxis of TB, including RIF (Granich et al. 2000, Singh $\&$ Kaur 2004). Prevalence rate of TB in 5 years ago in Iran were reported as high as 141 in 100,000 in Zabol (Khalil 2003 ) and as low as 11.9 in 100,000 in Isfahan (Zandeeh et al. 2003).

Because of its high bactericidal action, RIF, along with INH, forms the backbone of short-course chemotherapy. Resistance to RIF is increasing because of widespread application and results in selection of mutants resistant to other components of short-course chemotherapy; $88 \%$ of hospitalized patients with drug resistant TB in Massih Daneshvari Hospital (Iran-Tehran) were resistant to at least INH and RIF (Masjedi et al. 2003). In $56 \%$ the TB bacillus was resistant to all four drugs: INH, RIF, streptomycin (STM), and ethambutol (EMB); 85\% had resistance to STM at least and $60 \%$ were at least resistant to EMB. Most drug resistant cases of TB in were seen among Afghan emigrants; 95\% of cases had a history of treatment at least once, and the resistance was secondary; $36 \%$ of MTB strains isolated from patients in Mshhad-Iran were resistant to at least one anti-TB drug (Namaei et al. 2003). The highest rate of resistance was observed for SM: 34.29 and $60 \%$ of strains isolated from newly and previously treated patients respectively were resistant to streptomycin; 4\% were multidrug resistant (MDR). 
Characterization of the $r р о B$ gene (a region responsible to synthesis of beta-subunit of RNA polymerase) in Escherichia coli demonstrated that RIF specifically interacts with this subunit; thereby hindering transcription, and mutations in the rpoB locus conferred conformational changes leading to defective binding of the drug and consequently resistance (Jin \& Gross 1988). Several techniques were used for detecting these mutations and subsequently, the rроB locus from $M$. tuberculosis was characterized and mutations conferring the resistant trait were identified (Telenti et al. 1993a, Kapur et al. 1994).

Automated sequencing has been unambiguously applied to characterize mutations associated with resistance to RIF, but a number of other techniques such as PCRsingle-strand conformational polymorphism (PCR-SSCP), have been successfully applied (Pretorius et al. 1995, Morris et al. 1995, Kalia et al. 1997).

PCR-SSCP analysis, involves amplification of a segment of the gene encoding for the specific drug target and comparison of PCR products of drug-sensitive and drug-resistant strains by non-denaturing electrophoresis, in which mutations usually result in an altered pattern (Snider \& Roper 1992, Iseman 1993, Telenti et al. 1993b). However recent studies have questioned its sensitivity and specificity (Lee et al. 1998, Miriam et al. 2001).

Also, from various region of the world, different types of mutations were identified in RIFr M. tuberculosis clinical isolates. Most of these were point mutations involving 10 codons, while only few isolates had a deletion or insertion. Most frequently involved in mutation in the various regions of the world were codon 531 and codon 526 (Matsiota et al. 1998, Lee et al. 1998, Spindola et al. 2001, Miriam et al. 2001). In this study, we investigated the usefulness of PCR-SSCP method to detect mutations in the rpoB gene of RIFr and RIFs M. tuberculosis strains isolated in Iran from 2002 to 2004. Also the frequencies of mutations in their rрoB gene were characterized by direct sequencing to determine the codons most frequently involved in resistance to RIF in Iran.

\section{MATERIALS AND METHODS}

In the present study, 37 M. tuberculosis strains, originating from the Iranian Tuberculosis Center were used; they had been sent from laboratories and TB centers in Iran from 2002 to 2004. All isolates were characterized by conventional methods (Staining by Ziehl Neelsen method, observation of speed of growth and colony morphology and nitrate reduction test) and by specific amplification of the Direct Repeat (DR) gene region only present $M$. tuberculosis complex (Bahremand 1992, Tavakoli et al. 2005). The indirected agar proportion method test used for testing the resistance or sensitivity of pure cultures of M. tuberculosis isolates (Williams et al. 1998, Charley et al. 1999). All isolates were cultured in $7 \mathrm{H} 9$ broth medium for ten days. Subsequently, LJ medium without and with RIF were inoculated with $0.1 \mathrm{ml}$ of $10^{-2}$ and $10^{-4}$ dilutions of a McFarland 1.0 concentration of a suspension of each isolate of M. tuberculosis. The inoculated plates were then incubated at $37^{\circ} \mathrm{C}$ for three weeks. The break-point was $1 \%$. The isolate was considered susceptible to RIF if the number of colonies that grew on the drug-containing plates were more than $1 \%$ of the number of colonies that grew on the RIF-free control. An isolate was considered resistant if $1 \%$ or more grew on the drug-containing plate. Chromosomal DNA of 37 clinical isolates, 21 RIFr and 16 RIFs, were extracted by "DNA mini-prep" procedure using Hexadecyltrimethylam-moniumbromide (CTAB) as previously described (Cooper et al. 1999, Spindola et al. 2001). About 10 ng of extracted DNA was used for PCR amplification. The DNA of drug-susceptible standard strain, H37Rv (ATCC 27294) was used as control sample in this study.

PCR was performed as following: PCR reactions (50 $\mu 1)$ contained $10 \mathrm{ng}$ target DNA, 15 pmol primers, $2 \mathrm{mM}$ dNTP (Pharmacia Biotech), 2.5U Taq polymerase (MBI Fermentas), $1.5 \mathrm{mM} \mathrm{MgCl}_{2}$, and $5 \mu 110 \times$ buffer. The reaction was performed in a thermal cycler from PCR Hybid (Omnigene).

The sequence of primers for the $r p o B$ locus were (rpo 105) 5'-CGTGGAGGCGATCACACCGCAGACGT-3' and primer (rpo 273) 5'-GACCTCCAGCCCGGCACGCTCACG3', which produced a 193-bp amplicons (Spindola et al. 2001). Samples were then subjected to one cycle of $96^{\circ} \mathrm{C}$ for $5 \mathrm{~min}$, followed by 40 cycles of $96^{\circ} \mathrm{C}$ for $1 \mathrm{~min}, 55^{\circ} \mathrm{C}$ for $1 \mathrm{~min}, 72^{\circ} \mathrm{C}$ for $30 \mathrm{~s}$, and one final cycle of $72^{\circ} \mathrm{C}$ for $10 \mathrm{~min}$. PCR products were run on $2 \%$ agarose gel and examined for the presence of the 193-bp band after ethidium bromide staining (Travis 1995, Miriam et al. 2001).

The PCR products were SSCP analyzed by electrophoresis on $10 \%(\mathrm{w} / \mathrm{v})$ acrylamide gels. In brief, the SSCP gel was made by mixing $10 \mathrm{ml} 40 \%(\mathrm{w} / \mathrm{v})$ acrylamide solution, $25.6 \mathrm{ml} \mathrm{H} 2 \mathrm{O}, 4 \mathrm{ml} 10 \times$ TBE, $30 \mu \mathrm{T}$ TEMED, and $300 \mu \mathrm{l}$ ammonium persulfate. About $6 \mu \mathrm{l}$ of amplified product was mixed with $4 \mu \mathrm{l}$ of loading buffer ( $95 \%$ formamide, $20 \mathrm{mM}$ EDTA, and $0.05 \%$ bromophenol blue) and the mixtures was boiled for $4 \mathrm{~min}$, cooled on ice for $5 \mathrm{~min}$, and then loaded on the acrylamide gel at $2 \mathrm{~W}$ for $14-16 \mathrm{~h}$ in cold room. The gel was silver stained by using $10 \%(\mathrm{v} / \mathrm{v})$ gacial acetic acid as fix/stop solution; silver nitrate solution (contained $2 \mathrm{~g} \mathrm{AgNO}$, 21 ultrapure water and $3 \mathrm{ml} \mathrm{37 \%}$ formaldyde) as stain solution and solution contained $60 \mathrm{~g}$ sodium carbonate, 21 ultrapure water, $3 \mathrm{ml} 37 \%$ formaldyde and $400 \mu \mathrm{l}$ sodium thiosulfate as developing solution. SSCP assays were repeated at least two times with all isolates.

Twenty one samples of 193-bp rpoB amplicons with different PCR-SSCP patterns from RIFr (resistance) and 10 RIFs (sensitive) were sequenced. The products were sequenced by using upstream primer rpo 105 with Applied Biosystems 377 automated sequence protocol (ABI PRISM $^{\mathrm{TM}}$ Dye Terminator). All post runs analysis was performed using Clustalw, version 1.82, and software, EMBL-EBI. Each sequence was compared both with the control strain sequence and with the published rpoB sequence (GenBank accession NC 000962 REGION: 759805.763323).

\section{RESULTS}

The results of susceptibility testing indicated that percentage resistance were from less than 1 to 31 on LJ medium contained $4 \mu \mathrm{g}$ RIF ml$l^{-1}$. This percentage resistance were less than 1 for all sensitive isolates. Prepara- 
tions of inoculums were problematic, because of over- or under inoculation, so that we had to repeat the experiment for more than six times in about 8 months. The PCRSSCP patterns from strains is shown in Fig. 1. On the basis of the SSCP results, the 21 RIFr isolates were grouped in seven groups: pattern 1, 4, and 5 with 4 bands; pattern 2 and 3 with 5 bands; pattern 6 , similar to H37Rv; and pattern 7 with 3 bands. Of the 16 RIFs, 15 strains (93.8\%) had a PCR-SSCP pattern identical to H37Rv; and 1 strain $(6.2 \%)$ had different pattern. One of the resistant isolates had a PCR-SSCP pattern similar to that of M. tuberculosis susceptible control strain H37Rv and one of the RIFs strains had a PCR-SSCP pattern differ to that of susceptible control strain H37Rv.

After SSCP analysis, 21 PCR product samples from RIFr and 10 from RIFs were sequenced. An electropherograms of automatic DNA sequencing is shown in Fig. 2. Of the 21 sequences of RIFr isolates, 18 strains showed a single mutation in the 81-bp region of the $r p o B$ gene. Three strains with 1 to 5 -percentage resistance in $4 \mu \mathrm{g} \mathrm{RIF} \mathrm{ml}{ }^{-1}$ did not show any mutation within the 81-bp core region. $14.3 \%$ had mutations caused polymorphism and therefore should have another mutation not in the 81-bp region that needs to be characterized. Ten samples had a mutation at codon 531(Ser/Leu), four at codon 526 (His/Tyr), one at the codon 516 (Asp/Val). Three stains had polymorphism in codons, 523 (Gly/Gly), 512 (Ser/Ser), and 511 $(\mathrm{Leu} / \mathrm{Leu})$ with uncharacterized mutation in another region outside the 81-bp region and finally three strains did not have neither mutations nor polymorphism. These data are summarised in Table.

\section{DISCUSSION}

Most of resistant strains (95.2\%) showed PCR-SSCP patterns different from that of $\mathrm{H} 37 \mathrm{Rv} ; 28.6 \%$ had four bands and $23.8 \%$ had three bands.

These finding demonstrated that frequencies of particular PCR-SSCP patterns in RMP-resistant $M$. tuberculosis isolates from Iran have some differences from those that have been reported from other geographic areas (Kochi et al. 1993, Telenti et al. 1993a,b, Kim et al. 1997, 1999, Lee et al. 1998, Miriam et al. 2001). We repeated our experiments several times and the results demonstrated

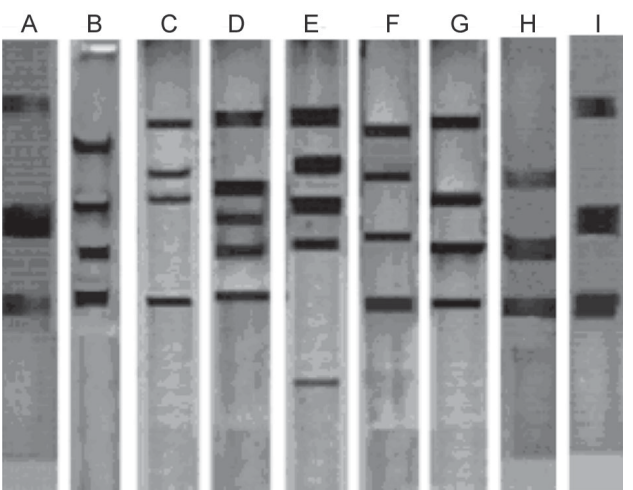

Fig. 1: nine different polymerase chain reaction-single-strand conformation polymorphism patterns including standard, rifampin resistant (RIFr) and sensitive (RIFs) strains observed in our study. A: H37Rv standard; B: a RIFs pattern differed from H37Rv and from $\mathrm{C}$ to $\mathrm{H}$ patterns observed in RIFr strains; I: a RIFr strain with pattern similar to H37Rv.

\section{TABLE}

Specific mutations identified in 21 rifampin resistance and one sensitive strains of Mycobacterium tuberculosis isolated from Iran

\begin{tabular}{lcc}
\hline $\begin{array}{l}\text { Mutated } \\
\text { rpoB codon } \\
\text { No mutation }\end{array}$ & $\begin{array}{c}\text { Specific } \\
\text { mutation }\end{array}$ & $\begin{array}{c}\text { No. of strains } \\
3 \text { resistant } \\
(14.3 \%)\end{array}$ \\
\hline 516 & 0 & 1 resistant \\
& GAC/GTC & $(4.75 \%)$ \\
523 & (Asp/Val) & 1 resistant \\
& GGG/GGT & $(4.75 \%)$ \\
526 & (Gly /Gly) & 4 resistant \\
& CAC/TAC & $(19.1 \%)$ \\
531 & (His/Tyr) & 10 resistant \\
& TCG/TTG & $(47.5 \%)$ \\
511 & (Ser/Leu) & 1 resistant \\
& CTG/TTG & $(4.75 \%)$ \\
512 & $($ Leu/Leu) & 1 resistant \\
& AGC/TGC & $(4.75 \%)$ \\
531 & (Ser/Ser) & 1 sensitive \\
& TCG/TTG & \\
\hline
\end{tabular}

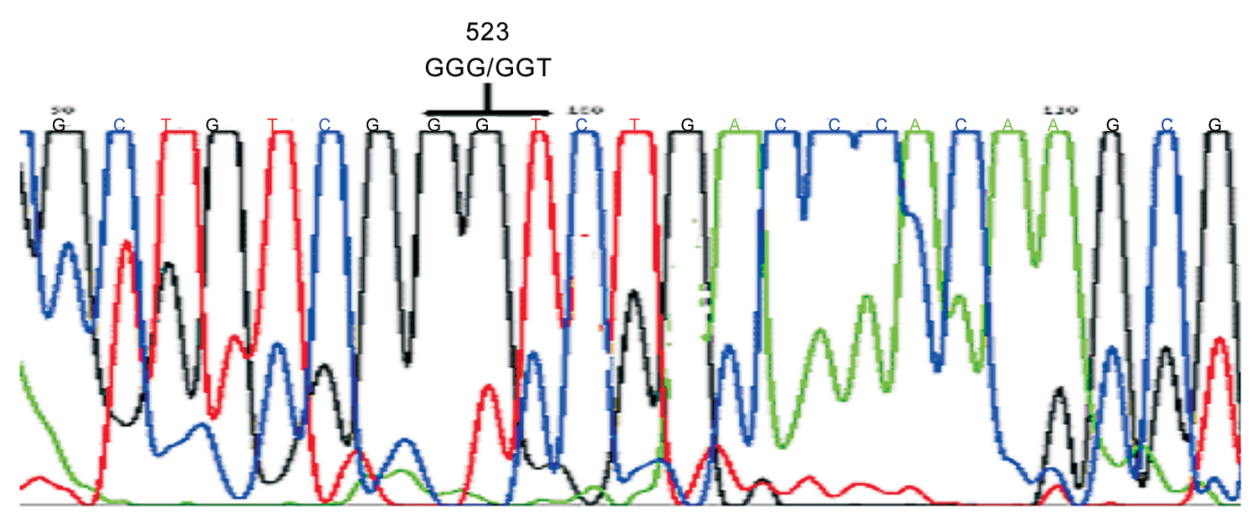

Fig. 2: electropherogram representative of part of the 193-bp region of $r p o B$ gene sequence, revealed a mutation at codon 523 [GGG/GGT (Gly/Gly)]. 
that our technique is highly reproducible; although it should be considered that differences in methods might produce different banding pattern.

Nonetheless local differences, our data also demonstrated that SSCP analysis is highly specific and sensitive for detecting mutations in the rpoB gene in RIFr $M$. tuberculosis. The sensitivity of this method was $95.2 \%$ with the specificity of $93.8 \%$. Thus this method can be considered as a convenient method for detection of mutations responsible for conferring RIFr.

Our results are the same as to those of Bahremand et al. (1998) finding but are not exactly conform to Spindola de Miranda et al. (2001), demonstrating that this method may perform poorly in detecting mutations in $r p o B$ region. However, in this study, $1 / 21$ (4.75\%) of the resistant isolates had a PCR-SSCP pattern similar to that of M. tuberculosis susceptible control strain H37Rv. These results may imply a mutation in another part of the $r p o B$ gene or the existence of at least one additional gene that participates in RIFr. Sequencing result of this strain demonstrated no mutation in the 81-bp region. Also 1/16 (6.3 $\%$ ) of the sensitive strains had a PCR-SSCP pattern different from that of susceptible control strain H37Rv that may imply the validity of proportion method is lower than $100 \%$. Surprisingly sequencing of this strain demonstrated codon 531 (TCG/TTG) mutation that cause (Ser/Leu) change.

A study on RIFr detection of M. tuberculosis from Brazilian patients using reverse-phase hybridization assay was performed by de Oliveira et al. (2003). They found that although all sensitive strains had the sensitive genotype, but among resistant isolates, a sensitive genotype was obtained in three due to the absence of mutations in the hot spot region, demonstrating an assay accuracy of $97.6 \%$ for detection of drug susceptibility. They also reported that, using specific probe for the mutation; the sensitivity to detect heteroresistance is similar to that of the proportion method.

Our PCR-SSCP analysis results are not fully consistent with that of reports from other geographic areas (Telenti et al. 1993b, Xiao-Yong et al. 2003, Tracevskr et al. 2002). On the basis of the SSCP results of Miriam et al. (2001), the 35 RIFr isolates were grouped in two main categories: group one, 24 isolates (68.6\%) with an SSCP identical to that of the control strain H37Rv, and group two, 11 isolates $(31.4 \%)$ with an SSCP different from that of H37Rv. In their study there also were 11 RIFs isolates that showed an SSCP identical to that of H37Rv. Lee et al. (1998) assessed the molecular mechanism of RIFr in clinical strains of M. tuberculosis. The molecular nature of a part of the rpoB gene in $77 \mathrm{M}$. tuberculosis clinical strains isolated in Korea was analyzed using PCR-SSCP and PCR-sequence analysis. Among 67 RIFr isolates, 50 showed SSCP profiles different from that of a RIFr control strain, $M$. tuberculosis $\mathrm{H} 37 \mathrm{Rv}$, while 17 resistant isolates displayed SSCP profiles indistinguishable from that of the sensitive control strain. This might be due to the presence of mutation outside of the amplicon.

After sequencing of 81-bp region of the $r p o B$ gene of 21 RIFr isolates, $85.7 \%$ strains showed a single mutation. An additional interesting finding was that three strains
(14.3\%) with 1 to 5 -percentage resistance in a MIC of $4 \mu \mathrm{g}$ RIF $\mathrm{ml}^{-1}$ did not show a mutation within the 81 -bp core region. This observation confirms a recent report of a RIFr strain identified in Australia with a mutation outside the core region of $r p o B$ (Lilly et al. 1999); $14.3 \%$ had mutations causing SSCP polymorphism, with a probable mutation in another region. Upon analysis of mutation frequency distribution, $47.6 \%$ had a mutation at codon 531 (Ser/Leu). In addition, 19\% isolates had mutations at codon 526 (His/Tyr) and 4.7\% at the codon 516 (Asp/Val) (Table). These finding is comparable to the results of early studies demonstrating the rрoB mutation frequencies in isolates from the United States (Kapur et al. 1994, Telenti et al. 1993a,b), European and African countries (Lilly et al. 1999, Hirano et al. 1999), Japan (Ohno et al. 1996, Suzuki et al. 1995), Brazil and France (Spindola et al. 2001) and Asian countries (Hirano et al. 1999). In summary, our data on rров mutation frequencies in isolates from Iran supported the common notion that rifampin resistance genotypes with mutations at critical codons, i.e., those encoding Ser531 and His 526 were the most frequently found in M. tuberculosis populations regardless of geographic origin.

Mutations associated with nucleotide replacements in codons 513 and 526 were associated with high-percentage RIFr, whereas mutations in codon 523 and 512 were observed in low-percentage RIFr. Other authors (Kapur et al. 1994) have reported high and low levels of resistance associated with specific nucleotide replacements. These differences reflect the complex and crucial interaction between the drug and its target at the molecular level, where the position of the affected allele seems to be critical.

\section{ACKNOWLEDGEMENTS}

To Dr Ghazisaeedi from Tehran University and Dr Karimi from Pasteur institute of Iran for scientific associations and providing mycobacterial strains.

\section{REFERENCES}

Ashok R, Awdhesh K, Nishat A 1998. Multidrug-resistant Mycobacterium tuberculosis: molecular perspectives. Emerg Infect Dis 4: 195-210.

Bahremand A 1992. Laboratory Procedures for the Isolation and Identification of Mycobacteria, Pastor Institute of Iran Press, Iran.

Bahremand AR, Velayati AA, Feizabadi MM, Sanami A, Babaei MR, Bakayev VV 1998. Rapid detection of resistance to rifampin in Mycobacterium tuberculosis by single strand conformation polymorphism. Arch Irn Med 1: 17-21.

Bloom BR, Murray C 1992. Tuberculosis: commentary on a reemergent killer. Science 257: 1055-1064.

Charley W, Mitchell M, Kidd K 1999. Antibiotics laboratory medicine. In The Antimycobacterial Susceptibility Tests, Williams \& Wilkins Press, New York, p. 127-175.

Cooper C, Revised by Jovell B 1999. Isolation and quantification of genomic DNA from Mycobacterium tuberculosis. WWW. Wadsworth. Org / rflp/ tutorials/ DNA isolation.html.

de Oliveira MM, da Silva Rocha A, Cardoso Oelemann M, Gomes HM, Fonseca L, Werneck-Barreto AM, Valim AM, Rossetti ML, Rossau R, Mijs W, Vanderborght B, Suffys P 
2003. Rapid detection of resistance against rifampicin in isolates of Mycobacterium tuberculosis from Brazilian patients using a reverse-phase hybridization assay. J Microbiol Method 53: 335-342.

Frieden TR, Sterling T, Pablos-Mendez A, Kilburn JO, Cauthen JO, Dooley SW 1993. The emergence of drug-resistant tuberculosis in New York City. N Engl J Med 328: 521-526.

Garcia-Garcia ML, Jimenez-Corona ME, Ponce-de-Leon A, Jimenez-Corona A, Palacios Martinez M, Balandrano-Campos S 2000. Mycobacterium tuberculosis drug resistance in a suburban community in southern Mexico. Int $J$ Tuberc Lung Dis 4: 168-170.

Granich R, Balandrano S, Santaella A, Binkin N, Castro K, Marquez-Fiol A 2000. Survey of drug resistance of Mycobacterium tuberculosis in 3 Mexican states. Arch Intern Med 160: 639-644.

Hirano K, Abe C, Takahashi M 1999. Mutations in the rpoB gene of rifampin-resistant Mycobacterium tuberculosis strains isolated mostly in Asian countries and their rapid detection by line probe assay. J Clin Microbiol 37: 26632666.

Iseman M 1993. Treatment of multidrug-resistant tuberculosis. N Engl J Med 329: 784-791.

Jin D, Gross C 1988. Mapping and sequencing of mutations in the Escherichia coli rpoB gene that leads to rifampin resistance. J Mol Biol 202: 45-58.

Kalia A, Ahmad N, Rattan A 1997. Diagnosis of multi-drug resistant tuberculosis: comparison of traditional, radiometric and molecular methods. In Abstracts of the 20th International Congress of Chemotherapy; International Society of Chemotherapy, Sydney, Australia, p. 211.

Kapur V, Li LL, Iordanescu S, Hamrick M, Wanger BN, Mooser JM 1994. Characterization by automated DNA sequencing of mutations in the gene $r p o B$ encoding the RNA polymerase B subunit in rifampin resistant Mycobacterium tuberculosis strains from New York City and Texas. J Clin Microbiol 32: 1095-1098.

Kato-Maeda M, Sifuentes-Osornio J, Bobadilla-del-Valle M, Ruiz-Palacios GM, Ponce-de-Leon A 1999. Drug resistance among acid-fast bacilli. Lancet 353: 1709-1715.

Khalili S 2003. Epidemiological evaluation of TB in Zabol district, 1998-2002. 17th National Congress of Tuberculosis, Isfahan, Iran, p. 48.

Kim BJ, Kim SY, Park BH, Lyu MA, Park IK, Bai GH, Kim SJ, Cha CY, Kook YH 1997. Mutations in the rpoB gene of Mycobacterium tuberculosis that interfere with PCR-SSCP analysis for rifampin susceptibility testing. J Clin Microbiol 35: 492-494.

Kim BJ, Lee SH, Lyu MA, Kim SJ, Bai GH, Kim SJ, Chae GT, Kim EC, Cha CY, Kook YH 1999. Identification of mycobacterial species by comparative sequence analysis of the RNA polymerase gene (rpoB). J Clin Microbiol 37: 17141720.

Kochi A, Vareldzis B, Styblo K 1993. Multi-drug resistant tuberculosis and control. Res Microbiol 144: 104-110.

Lee H, Cho S, Bang H, Lee J, Bae G, Kim S 1998. Molecular analysis of rifampin-resistant Mycobacterium tuberculosis isolated from Korea by polymerase chain reaction-single strand conformation polymorphism sequence analysis. Int
J Tuberc Lung Dis 2: 585-589.

Lilly K, Yuen W, David L, Peter JC 1999. Bacteriological and molecular analysis of rifampin-resistant Mycobacterium tuberculosis strains isolated in Australia. J Clin Microbiol 37: 3844-3850.

Masjedi MR, Amiri MV, Mirsaiedi SM, Mohajer K, Mansouri SD, Velayati AA 2003. Tuberculosis bacillus drug resistance in hospitalized patients with drug resistant tuberculosis in Massih Daneshvari Hospital. 17th National Congress of Tuberculosis, Isfahan, Iran, p. 33.

Matsiota-Bernard P, Vrioni G, Marinis E 1998. Characterization of rpoB mutations in rifampin-resistant clinical Mycobacterium tuberculosis isolates from Greece. J Clin Microbiol 36: 20-23.

Miriam BV, Alfredo PL, Catalina AH, Gilberto VA, Midori KM, Peter MS, Patricia C, Guillermo MR, Jose SO 2001. rpoB gene mutations in rifampin-resistant Mycobacterium tuberculosis identified by polymerase chain reaction singlestranded conformational polymorphism. Emerging Infectious Disease 7: 1010-1013.

Morris S, Bai G, Suffys P, Portillo-Gomez L, Fairchok M, Rouse D 1995. Molecular mechanisms of multidrug resistance in clinical isolates of Mycobacterium tuberculosis. $J$ Infect Dis 171: 954-960.

Namaei MH, Nazem M, Sadeghian A, Naderinasab M 2003. Drug resistance of Mycobacterium tuberculosis strains isolated from patients with tuberculosis in Mashhad, Iran. 17th National Congress of Tuberculosis, Isfahan, Iran, p. 41.

Ohno H, Koga H, Kohno S, Tashiro T, Hara K 1996. Relationship between rifampin MICs for and rpoB mutations of Mycobacterium tuberculosis strains isolated in Japan. Antimicrob Agents Chemother 40: 1053-1056.

Pretorius G, Van Helden P, Sergel F, Eisenach K, Victor T 1995. Mutation in katG gene sequences in isoniazid-resistant clinical isolates of Mycobacterium tuberculosis are rare. Antimicrob Agents Chemother 39: 2276-2281.

Singh S, Kaur M 2004. High rate of primary and acquired drug resistance in India isolated Mycobacterium tuberculosis. Inter J Infect Dis 8: 255-260.

Snider D, Roper WL 1992. The new tuberculosis. NEngl J Med 326: 703-705.

Spindola de Miranda S, Kritski A, Filliol I, Mabilat C, Panteix $\mathrm{G}$, Drouet E 2001. Mutations in the rpoB gene of rifampin resistant Mycobacterium tuberculosis strains isolated in Brazil and France. Mem Inst Oswaldo Cruz 96: 247-250.

Suzuki Y, Katsukawa C, Inoue K, Yin Y. P, Tasaka H, Ueda N, Makino M 1995. Mutations in rpoB gene of rifampicin resistant clinical isolates of Mycobacterium tuberculosis in Japan. J Jpn Assoc Infect Dis 69: 413-419.

Tavakoli A, Radi E, Tamizifar H, Pishva E, Salehi R 2005. Molecular fingerprinting of Mycobacterium tuberculosis isolates from patients in Isfahan province using spoligotyping method. J Isf Med Sch 76-77: 108-114

Telenti A, Imboden P, Marchesi F, Lowrie D, Cole S, Colston M 1993a. Detection of rifampin-resistance mutations in Mycobacterium tuberculosis. Lancet 341: 647-650.

Telenti A, Imboden P, Marchesi F, Schmidheini T, Bodmer T 
1993b. Direct, automated detection of rifampin-resistant Mycobacterium tuberculosis by polymerase chain reaction and single-strand conformation polymorphism analysis. Antimicrob Agents Chemother 37: 2054-2058.

Tracevskr T, Jansone I, Broka L, Marga O, \& Baumanis V 2002. Mutation in rpoB and katG genes leading to drug resistance in Mycobacterium tuberculosis in Latvia. J Clin Microbiol 40: 3789-3792.

Travis G 1995. Step-by-Step SSCP. Laboratory of molecular systematic smithsonian institution, Washington, 20560. http://www.uga.edu/srel/DNA_Lab/SSCP'96V2.rtf

Xiao-Yong F, Zhong-Yi H, Fan-Hong X, Zhi-Qiang Y, Sheng-Qi
G, Zhong-Ming Li 2003. Rapid detection of rpoB gene in Mycobacterium tuberculosis isolated in Shanghai by using the amplification refractory mutation system. $J$ Clin Microbiol 41: 993-997.

Williams DL, Spring L, Collins L, Miller LP, Heifets LB, Gangadharam PRJ, Gillis TP, 1998. Contribution of rpoB mutations to development of rifamycin cross-resistance in Mycobacterium tuberculosis. Antimicrob Agents Chemother 42: 1853-1857.

Zandeeh M, Fareed F, Fadaee R, Moeenzadeh F 2003. Epidemiological evaluation of TB in Isfahan district, 1998-2002. 17th National Congress of Tuberculosis, Isfahan, Iran, p. 29. 